\title{
Clinical Evaluation of a New High-Sensitivity Cardiac Troponin I Assay for Diagnosis and Risk Assessment of Patients with Suspected Acute Myocardial Infarction
}

\author{
Masayuki Shiozaki $^{a}$ Kenji Inoue $^{\mathrm{a}}$ Satoru Suwa $^{\mathrm{b}}$ Chien-Chang Lee $^{c}$ \\ Shuo-Ju Chiang ${ }^{d}$ Akihiro Sato $^{a}$ Kentaro Fukuda $^{a}$ Naozumi Kubota $^{a}$ \\ Hiroshi Tamura ${ }^{a}$ Yasumasa Fujiwara $^{a}$ Kentaro Yamaguchi ${ }^{\mathrm{e}}$ Tomoaki Sato $^{\mathrm{e}}$ \\ Masataka Sumiyoshi ${ }^{a}$ Hiroyuki Daida $^{f}$ \\ aDepartment of Cardiology, Juntendo University Nerima Hospital, Tokyo, Japan; ${ }^{\circ}$ Department of Cardiology, \\ Juntendo University Shizuoka Hospital, Shizuoka, Japan; ' Department of Emergency Medicine, National Taiwan \\ University Hospital, Taipei, Taiwan; dDivision of Cardiology, Department of Internal Medicine, Taipei City Hospital

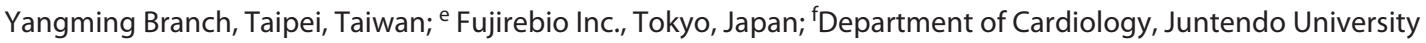 \\ School of Medicine, Tokyo, Japan
}

\section{Keywords}

Non-ST-elevation myocardial infarction $\cdot$ High-sensitivity cardiac troponin $\mathrm{T} \cdot$ High-sensitivity cardiac troponin I · Risk stratification · Asian

\begin{abstract}
Introduction: Current assays based on the 0-hour/1-hour (0-/1-h) algorithm using high-sensitivity cardiac troponin (hs-cTn) are limited to only Abbott Architect hs-cTnl, Siemens Vista hs-cTnl, and Roche Elecsys hs-cTnT. Objective: This study aimed to evaluate this new hs-cTnl assay, LumipulsePresto hs Troponin I, for diagnosis of acute myocardial infarction (AMI) on admission and on 0-/1-h algorithm to stratify AMI patients precisely. Methods: This prospective cohort study included 442 patients with suspected non-ST-elevation myocardial infarction in three hospitals in Japan and Taiwan from June 2016 to January 2019. We enrolled pa-
\end{abstract}

karger@karger.com www.karger.com/crd

Karger $\stackrel{\text { ' }}{5}$

GOPEN ACCESS
(C) 2021 The Author(s)

Published by S. Karger AG, Basel

This is an Open Access article licensed under the Creative Commons Attribution-NonCommercial-4.0 International License (CC BY-NC) (http://www.karger.com/Services/OpenAccessLicense), applicable to the online version of the article only. Usage and distribution for commercial purposes requires written permission. tients presenting to the emergency department with symptoms suggestive of AMI and collected blood samples on admission and 1 hour later. Two independent cardiologists centrally adjudicated final diagnoses; all clinical information was reviewed twice: first, using serial hs-cTnT (Roche-Elecsys, primary analysis) and Lumipulse Presto Lumipulse Presto, second, using the Lumipulse Presto hs-cTnl measurements. At first, we compared diagnostic accuracy quantified using receiver operating characteristic (ROC) curves for AMI. Then, we evaluated major adverse cardiovascular events (cardiac death, AMI) in the rule-out group according to a 0-hour/1-hour algorithm at the 30-day follow-up. Results: Diagnostic accuracy at presentation by the ROC curve for AMI was very high and similar for the LumipulsePresto hscTnland hs-cTnT, (area under the curve [AUC]:LumipulsePresto hs-cTnl, 0.89, 95\% confidence interval [Cl] 0.86-0.93; hscTnT, $0.89,95 \% \mathrm{Cl} 0.85-0.93 ; p=0.82$ ). In early presenters, the LumipulsePresto hs-cTnl appeared to maintain the diag- 
nostic performance of hs-cTn for patients with $<3 \mathrm{~h}$ (AUC: LumipulsePresto hs-cTnl, $0.87,95 \% \mathrm{Cl} 0.81-0.92$; hs-cTnT, $0.86,95 \% \mathrm{Cl} 0.80-0.92 ; p=0.81$ ). The algorithm using the LumipulsePresto hs-cTnl ruled out AMI in 200 patients with negative predictive value and sensitivity of $100 \%(95 \% \mathrm{Cl}$ $97.3 \%-100 \%)$ and $100 \%(95 \% \mathrm{Cl} 92.7 \%-100 \%)$, respectively, in the rule-out group. Conclusion: Diagnostic accuracy and clinical utility of the novel LumipulsePresto hs-cTnl assay are high and comparable with the established hs-cTn assays.

(C) 2021 The Author(s)

Published by S. Karger AG, Basel

\section{Introduction}

Chest pain is frequently encountered in the emergency department, and despite a decade of research, more than $2 \%$ of acute coronary syndrome cases are still missed or misdiagnosed [1,2]. Clinical assessment, 12-lead electrocardiography, and cardiac troponin are important diagnostic tools for early diagnosis of acute coronary syndrome in the emergency department. Especially, cardiac troponin levels have become the mainstay for acute coronary syndrome [3]. In 2015, the European Society of Cardiology (ESC) recommended early risk stratification for acute myocardial infarction (AMI) using the 0-hour/1-hour (0-/1-h) algorithm with high-sensitivity cardiac troponin (hs-cTn), which has been proposed as class A and evidence level B [4]. However, hs-cTn assay systems are limited to only Abbott Architect hs-cTnI, Siemens Vista hs-cTnI, and Roche Elecsys hs-cTnT. In the last 20 years, the definition of high-sensitivity system has been revised with an updated version published in 2018 [5]. This revision requires the improvement of the troponin assay system. In response to this, the International Federation of Clinical Chemistry (IFCC) defined high-sensitivity assay as follows: (1) the 99th percentile for hs-cTn assays should be determined in a healthy population of at least more than 300 male and female subjects; (2) the 99th percentile for hs-cTn assays should be measured with an analytical imprecision of $\leq 10 \%$ of the coefficient of variation (CV); and (3) the assays should measure cardiac troponin above the limit of detection in $\geq 50 \%$ of healthy subjects. Therefore, a new assay system for measurement of cardiac troponin should satisfy the suggestion of IFCC [6]. Recently, LumipulsePresto hs Troponin I (Fujirebio Inc., Japan) that meets the criteria of IFCC was developed. Thus, we aimed to evaluate this new hs-cTnI assay, LumipulsePresto hs Troponin I, for diagnosis of AMI on admission and on 0-/1-h algorithm to stratify AMI patients precisely.

New High-Sensitivity Cardiac Troponin I

Assay Using 0-h/1-h Algorithm

\section{Materials and Methods}

\section{Study Design and Patients}

This was a secondary analysis of a cohort study, titled "Diagnostics and Reduction of Asian Patients with Acute coronary syndrome Cost Analysis Base on the 0-/1-h algorithm using Highsensitivity cardiac Troponin (DROP-ACS) (UMIN000030668)," which is an ongoing prospective international multicenter diagnostic study being conducted at four sites in two countries (Japan and Taiwan) since November 2014. In this study, we measured samples from three hospitals because one hospital did not keep the sample in a deep freezer. Inclusion criteria were adults aged 30-89 years presenting cardiac symptoms from the American Heart Association (AHA) case definitions, namely, presence of acute chest, epigastric, neck, jaw, or arm pain or discomfort or pressure without apparent non-cardiac source [7]. Exclusion criteria were as follows: (1) ST-elevation myocardial infarction; (2) congestive heart failure, tachyarrhythmia, chronic kidney disease (serum creatinine level $>3 \mathrm{mg} / \mathrm{dL}$ ), pulmonary embolism, aortic dissection, or infectious disease (which increases troponin level) according to the results of screening examination on admission, such as blood tests, chest radiography, or ultrasonography. Finally, a total of 442 patients were analyzed in the study (online suppl. Fig. 1; for all online suppl. material, see www.karger.com/doi/10.1159/000512185; Table 1).

Informed consent was obtained from all patients. The study was approved by the local ethics committees and conducted in accordance with the Helsinki Declaration of 1971, as revised in 1982.

\section{Clinical Assessment}

All patients underwent an initial clinical assessment that included physical examination, clinical history, smoking habits (current, former smoker, and non-smoker), electrocardiography, blood pressure, heart rate, risk scores (Global Registry of Acute Coronary Events [GRACE]; Thrombolysis in Myocardial Infarction [TIMI]; or History, Electrocardiogram, Age, Risk Factors, Troponin [HEART]), and standard blood measurements.

\section{Adjudicated Final Diagnosis}

All attending physicians made a tentative diagnosis according to the algorithm, and the diagnosis was further confirmed at the 30-day follow-up using cardiac stress electrocardiography, stress scintigraphy, coronary computed tomography angiography, or coronary angiography. Two independent cardiologists reviewed all available medical records. AMI was defined according to the Fourth Universal Definition of Myocardial Infarction requiring evidence of myocardial necrosis together with ischemia (at least one of the electrocardiographic changes or positive findings of stress electrocardiography, myocardial perfusion scan, coronary computed tomography angiography, or coronary angiography during catheterization) [5]. Necrosis was diagnosed based on a rise or fall of cardiac troponin using the hs-cTnT assay system (Roche Diagnostics 5th generation) concentration with at least one value above the 99th percentile of the normal reference range at a level of assay imprecision near 10\% [8]. Type 1 myocardial infarction was characterized by atherosclerotic plaque rupture, ulceration, erosion, or dissection, with the resulting intraluminal thrombus in one or more coronary arteries leading to myocardial necrosis (any hs-cTnT concentration $>14 \mathrm{ng} / \mathrm{L}$, with rise and fall in hs-cTnT concentration where serial testing was available). 
Table 1. Baseline characteristics of the patients

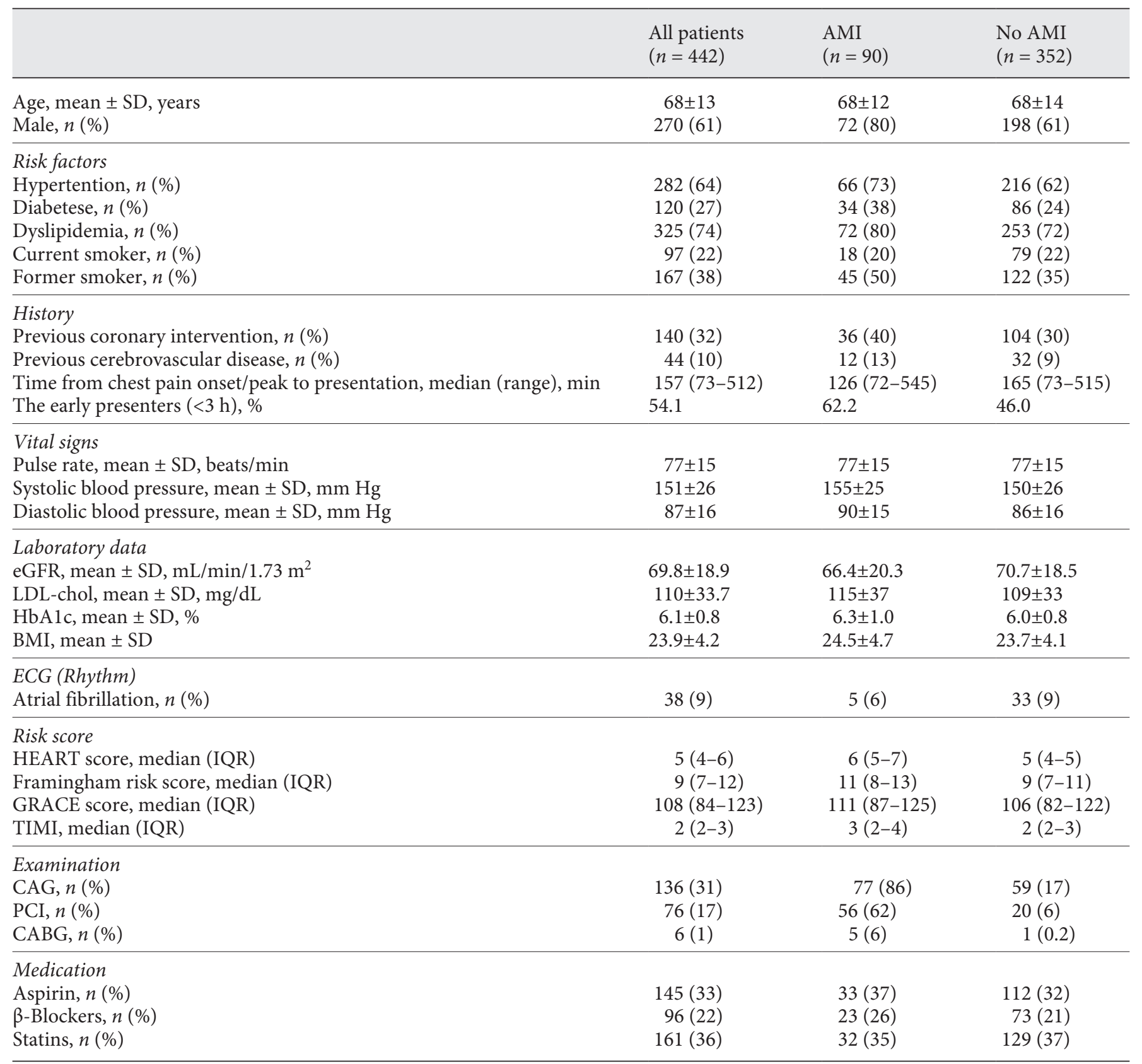

Type 2 myocardial infarction was defined as myocardial necrosis in which a condition other than coronary plaque instability contributed to an imbalance between myocardial oxygen supply and demand (e.g., coronary artery spasm, coronary embolisms, hypertension, or hypotension). All other patients were classified as "No AMI" in this analysis. Unstable angina was diagnosed in patients with normal hs-cTnT levels both on admission and 1-h follow-up in case of cardiac catheterization with severe stenotic lesion (>70\% stenosis) or clear appearance of thrombus in the culprit lesion in the coronary artery [4]. Atypical chest pain was diagnosed on the basis of the absence of findings from laboratory tests, electrocardiography, and chest radiography at the 30-day follow-up $[9,10]$.

\section{hs-cTnI System}

The new hs-cTnI assay, LumipulsePresto hs Troponin I, has a two-step sandwich assay principle with a fully automated chemiluminescent enzyme immunoassay system for LUMIPULSE L2400 or LUMIPULSE Presto II analyzer that can measure troponin I using serum and plasma. The capability of the assay system is pub- 


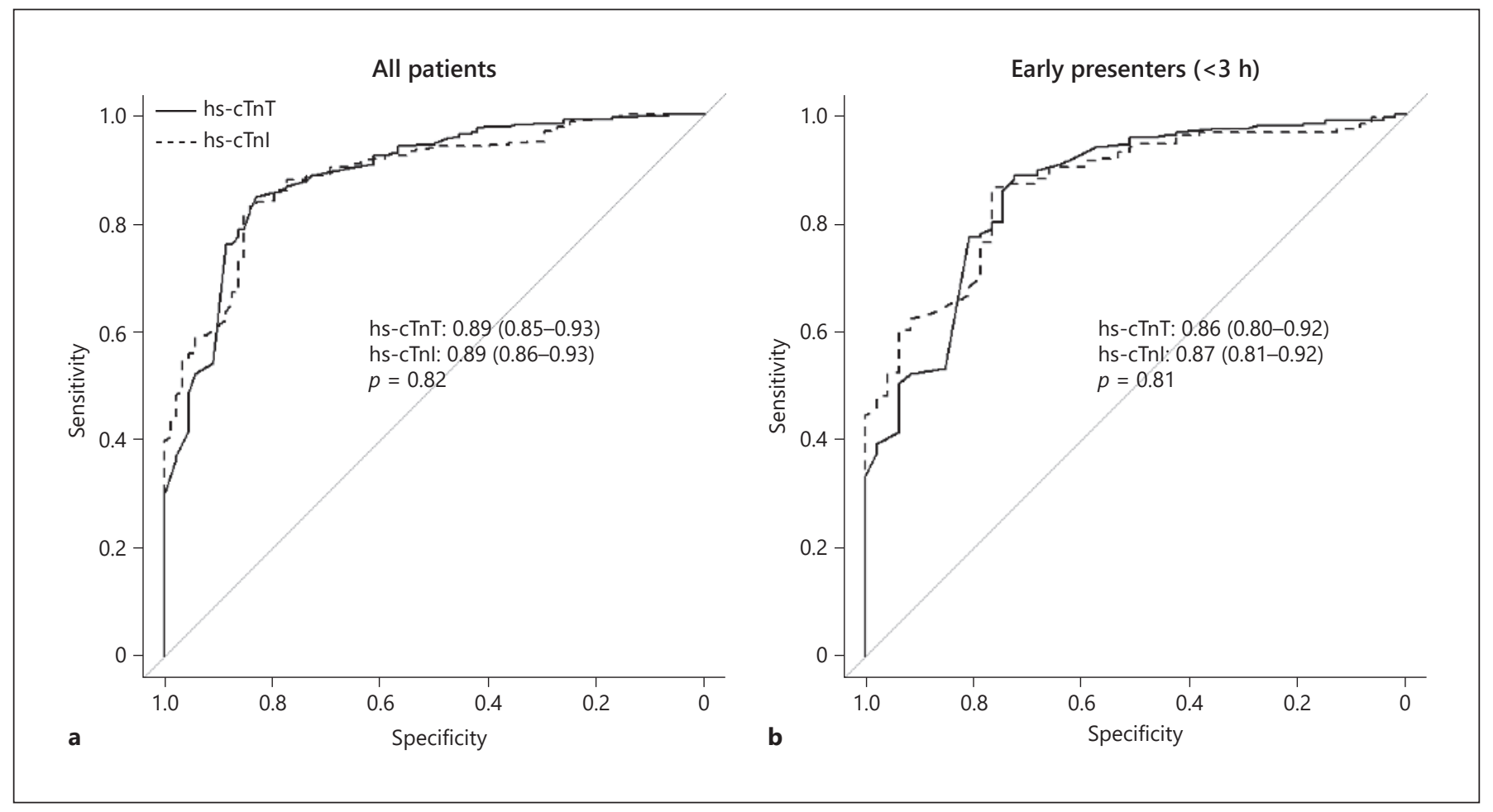

Fig. 1. Diagnostic performance of high-sensitivity cardiac troponin T and I. Receiver operating characteristic curves show the diagnostic accuracy of high-sensitivity cardiac troponin I and T for patients with non-ST-elevation myocardial infarction who presented to the emergency department with chest pain (a) and patients with chest pain onset within $3 \mathrm{~h} \mathrm{(b).}$

lished elsewhere [11]. We compared LUMIPULSE and Architect using patients' residual serum $(n=146$, including 104 samples with very low concentration of troponin I; less than $5 \mathrm{ng} / \mathrm{L}$ ) from the University of Tokyo Hospital (online suppl. Fig. 2).

\section{ESC 0-/1-h Algorithm}

The hs-cTn 0-/1-h algorithm uses hs-cTn blood concentrations at presentation and their absolute changes within $1 \mathrm{~h}$ to classify patients in the rule-out, observe, or rule-in group [4].

\section{End Points}

The primary end points were major cardiovascular events, including death and AMI at the 30-day follow-up in the "rule-out" group. Basically, all patients were seen by a cardiologist in the same hospital; however, if they failed to return for follow-up, they were tracked for adverse events at 30 days from the initial attendance shown on their hospital records and by telephone follow-up.

\section{Statistical Analysis}

Continuous variables are presented as mean [ \pm standard deviation (SD)] or median (interquartile range) and categorical variables as numbers and percentages. Receiver operating characteristic (ROC) curves was constructed to assess the sensitivity and specificity of both assays to compare their capability to diagnose AMI using hs-cTn data on admission. Diagnostic accuracy was reported as the area under the ROC curve and their corresponding 95\% confidence interval (CI). Based on their distribution, continuous variables were compared using one-way analysis of variance for three group comparisons. A $p$ value less than 0.05 was assessed as significant. The sensitivity and negative predictive value (NPV) for AMI in the rule-out group and the specificity and positive predictive value (PPV) for AMI in the rule-in group were calculated. Statistical analysis was performed using SPSS version 16.0 (SPSS Inc., Chicago, IL, USA), R (version 3.5.0; R Foundation for Statistical Computing, Vienna, Austria), and JMP version 9.0.0 (SAS Institute Inc., Cary, NC).

\section{Results}

\section{Patient Characteristics}

The baseline characteristics of 442 patients (mean age, 68 years; male, 61\%) with suspected non-ST-elevation myocardial infarction are shown in Table 1 . The median time from onset of chest pain to presentation was $157 \mathrm{~min}$ (lower quartile $73 \mathrm{~min}$, upper quartile $512 \mathrm{~min}$ ); in $54.1 \%$ of the patients, the time from onset of chest pain to presentation was $<3 \mathrm{~h}$. According to the 0 -/1-h algorithm, 128 early presenters were in rule-out group, 55 patients 
Fig. 2. European Society of Cardiology algorithm for high-sensitivity troponin $\mathrm{T}$ (a) and I (b). AMI, acute myocardial infarction; NSTEMI, non-ST-elevation myocardial infarction.

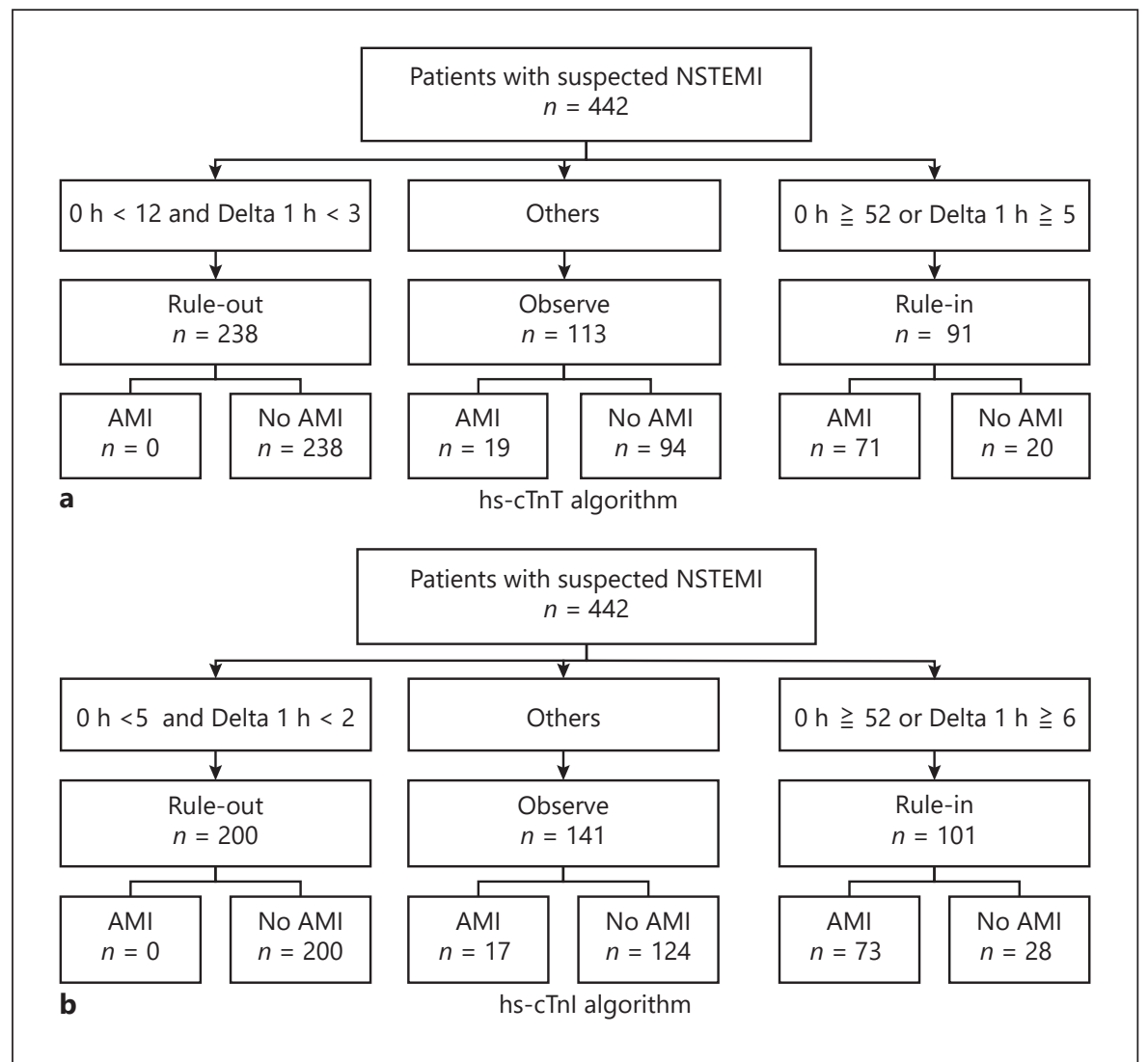

Table 2. Statistical analysis summary

\begin{tabular}{lcc}
\hline & hs-cTnT & hs-cTnI \\
\hline Sensitivity (95\% CI) & $100 \%(92.5-100)$ & $100 \%(92.7-100)$ \\
Specificity (95\% CI) & $92.2 \%(88.3-95.2)$ & $87.7 \%(82.7-91.7)$ \\
PPV (95\% CI) & $78.0 \%(68.1-86.0)$ & $72.3 \%(62.5-80.7)$ \\
NPV (95\% CI) & $100 \%(97.7-100)$ & $100 \%(97.3-100)$ \\
Accuracy & $93.9 \%(90.8-96.2)$ & $90.7 \%(86.8-93.7)$ \\
\hline
\end{tabular}

CI, confidence interval; hs-cTnT, high-sensitivity cardiac troponin T; hs-cTnI, high-sensitivity cardiac troponin I; NPV, negative predictive value; PPV, positive predictive value.

were in the observe group, and 56 patients were in the rule-in group.

In $62.2 \%$ of AMI patients, the time from onset of chest pain to presentation was $<3 \mathrm{~h}$. The adjudicated final diagnosis was AMI in 90 patients (20.4\%; type 1 myocardial infarction in 71 patients [16.1\%], type 2 myocardial infarction in 19 patients [4.3\%]), unstable angina in 15 patients (3.4\%), and symptoms of unknown origin (atypical chest pain) in 283 patients $(64.0 \%)$.
Diagnostic Performance of hs-cTnI and T for AMI at

Presentation according to Final Diagnoses

Overall, no difference in the diagnostic accuracy of AMI was found between hs-cTnT and hs-cTnI assay system on admission ROC analysis (area under the curve [AUC]: hs-cTnT, 0.89, 95\% CI 0.85-0.93; hs-cTnI, 0.89, 95\% CI $0.86-0.93$; $p=0.82$; Fig. 1). Dividing the cohort according to the duration of chest pain showed that the areas under the ROC curve appears to maintain the diagnostic performance of hs-cTn for patients with $<3 \mathrm{~h}$ (hscTnT 0.86, 95\% CI 0.80-0.92; hs-cTnI 0.87, 95\% CI 0.81$0.92 ; p=0.81$; Fig. 1). Table 2 describes the performance of the $0-/ 1$-h algorithm using the hs-cTn assay.

\section{Diagnostic Performance of hs-cTn Concentrations}

Measured according to the ESC 0-/1-h Algorithm

Figure 2 reveals the result of the 0 -/ 1 -h algorithm using hs-cTnT and hs-cTnI. The hs-cTnI algorithm ruled out significantly less patients than the hs-cTnT algorithm, i.e., $45 \%$ versus $54 \%$ (Fig. 3); however, none of the patients were diagnosed with AMI. Using the hs-cTnT algorithm, two patients in the rule-in group were stratified into the rule-out group; however, one patient underwent 


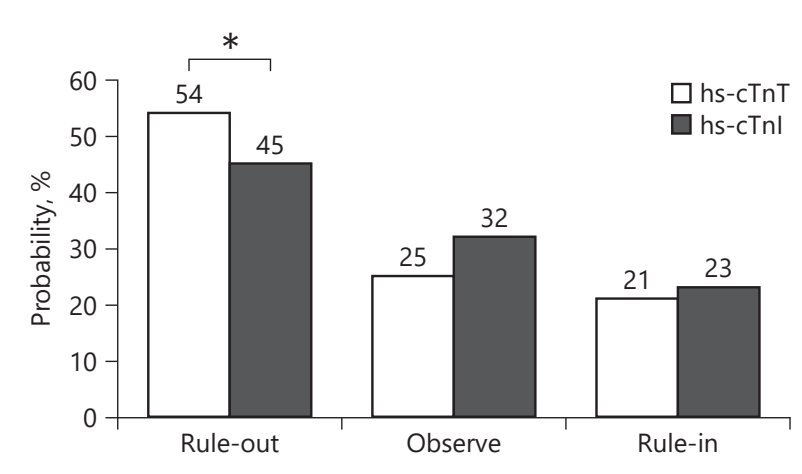

Fig. 3. Distribution pattern of patients suspected of non-ST-elevation myocardial infarction according to European Society of Cardiology algorithm. High-sensitivity troponin T, white square; high-sensitivity troponin I, gray square. ${ }^{*} p<0.05$.

coronary angiography which revealed moderate coronary restenosis (instantaneous wave-free ratio of 0.91 ), and another patient underwent coronary computed tomography angiography which revealed mild coronary artery disease. On the contrary, using the hs-cTnT algorithm, six patients in the rule-out group were stratified into the rule-in group. None of the patients had major adverse cardiovascular events or underwent percutaneous coronary intervention/coronary artery bypass grafting in the 30-day follow-up period. In the rule-in group for AMI, similar proportion of AMI was observed in both assay systems (hs-cTnI $[n=101 ; 23 \%]$ and hs-cTnT [ $n=$ 91; 21\%]). The specificity, PPV, and accuracy for AMI within each rule-in group were also similar at $87.7 \%$ (95\% CI $82.7 \%-91.7 \%$ ), $72.3 \%$ (95\% CI 62.5\%-80.7\%), and 90.7\% (86.8-93.7) for hs-cTnI compared with 92.2\% (95\% CI 88.3-95.2\%), 78.0\% (95\% CI 68.1-86.0\%), and 93.9\% (90.8-96.2) for hs-cTnT, respectively (Table 2).

\section{Discussion/Conclusion}

In this study, we evaluated whether the new hs-cTnI assay system, LumipulsePresto hs-cTnI, is useful for the diagnosis of AMI on admission and risk stratification using the 0-/1-h algorithm. This international multicenter cohort is prospectively developed for AMI based on the universal definition using hs-cTnT assay system, which has been recognized by the Food Drug Administration in the USA. LumipulsePresto hs-cTnI satisfied the criteria of IFCC as a high-sensitivity system [6]. In fact, some differences were found between hs-cTnI and hs-cTnT, in-

New High-Sensitivity Cardiac Troponin I

Assay Using 0-h/1-h Algorithm cluding their cutoff level as in previous reports [12, 13]; however, their diagnostic accuracy was comparable (AUC: hs-cTnT 0.89 ; 95\% CI $0.85-0.93$ vs. hs-cTnI 0.89; 95\% CI $0.86-0.93 ; p=0.82$ ) even in early presenters (<3 h: AUC [hs-cTnT] 0.86; 95\% CI $0.80-0.92$ versus AUC [hs-cTnI] 0.87; 95\% CI 0.81-0.92; $p=0.81)$. The clinical performance applied into the $0-/ 1$ - $h$ algorithm was also similar with that of the hs-cTnT assay, in terms of the stratification of patients suspected of non-STelevation myocardial infarction. The NPV and sensitivity for AMI in the rule-out group were $100 \%$, similar to the hs-cTnT algorithm. The hs-cTnI algorithm assigned $45 \%$ of the patients to the rule-out group, which was significantly small compared with the hs-cTnT algorithm. As a result, the proportions of the observe group and rule-in group were increased. This finding was similar with those in previous reports [14]. LumipulsePresto hs-cTnI has been developed using monoclonal antibodies that recognize the same epitopes with the Abbott ARCHITECT hs-cTnI assays; therefore, the LumipulsePresto hs-cTnI was considered to have characteristics similar to the Abbott ARCHITECT hs-cTnI assays. Small differences were noted in groups stratified by hs-cTnI and hs-cTnT. Six patients were stratified into the rule-in group according to hs-cTnI algorithm; however, no patients had AMI, and none underwent coronary angiography/percutaneous coronary intervention in the 30-day follow-up. On the contrary, according to the hs-cTnT algorithm, two patients in the rule-in group were stratified into the rule-out group correctly. These differences did not cause a problem in the risk stratification because no patients had AMI in the rule-out group as we mentioned earlier.

This study had several limitations. First, this study had a moderate cohort size and was a post-hoc analysis of a prospective trial. Second, we excluded patients with chronic kidney disease (serum creatinine level $>3.0 \mathrm{mg} /$ $\mathrm{dL}$ ) because chronic kidney disease itself is a high risk for acute coronary syndrome, and this algorithm could not have been suitable for such patients. Third, we excluded patients with congestive heart failure and tachyarrhythmia, which cause an increase in the cardiac troponin level because of the oxygen supply/demand imbalance, but not atherosclerotic plaque disruption with thrombosis. This study was carried out to evaluate a new hs-cTnI system for patients with ischemic heart disease. To avoid a bias, we excluded the aforementioned group. How to manage the patients with type II myocardial infarction is a different topic and needs further investigation.

In conclusion, the LumipulsePresto hs-cTnI assay system has similar capability with the hs-cTnT system. 
Moreover, this assay system could be applied to the 0-1 1-h algorithm and help identify patients who can return home safely.

\section{Acknowledgement}

We thank the staff in EDs and technicians (particularly Maki Kudo and Rie Nomura).

\section{Statement of Ethics}

The study was approved by the local ethics committees and conducted in accordance with the Helsinki Declaration of 1971, as revised in 1982. Informed consent was obtained from all patients.

\section{Conflict of Interest Statement}

Dr. Inoue has received research grant from Grant-in-Aid for Scientific Research (C), Roche Diagnostics, S.B. Bioscience Co., Ltd, Fujirebio Inc., Bayel and Kanto chemical Co., Inc. Dr. Daida has received research grant from Public Health Research Foundation, Actelion Pharmaceuticals Japan, Otsuka Parmaceuticals Co, Ltd, Nihon Medi-physics Co., Ltd., Teijin Parma, HeartFlo Japan GK, Novo Nordisk, Sumitomo- Dainippon Co, Ltd, Fukuda Denshi, ResMed, Philips Japan. Other authors report no relationships that could be construed as conflicts of interest.

\section{Funding Sources}

This work was supported by Japanese KAKENHI Grant Number JP18K09954.

\section{Author Contributions}

Design, analysis, interpretation of data; Kenji Inoue, Masayuki Shiozaki, Masataka Sumiyoshi, Hiroyuki Daida. Interpretation of data; Masayuki Shiozaki, Kenji Inoue, Tomoaki Sato, Kentaro Yamaguchi. Data collecting; Masayuki Shiozaki, Satoru Suwa, ChienChang Lee, Shuo-Ju Chiang, Akihiro Sato, Kentaro Fukuda, Naozumi Kubota, Hiroshi Tamura, Yasumasa Fujiwara.

\section{References}

1 Pope JH, Aufderheide TP, Ruthazer R, Woolard RH, Feldman JA, Beshansky JR, et al. Missed diagnoses of acute cardiac ischemia in the emergency department. N Engl J Med. 2000 Apr;342(16):1163-70.

2 Neumann JT, Sörensen NA, Schwemer T, Ojeda F, Bourry R, Sciacca V, et al. Diagnosis of myocardial infarction using a high-sensitivity troponin I 1-hour algorithm. JAMA Cardiol. 2016 Jul;1(4):397-404.

3 Amsterdam EA, Kirk JD, Bluemke DA, Diercks D, Farkouh ME, Garvey JL, et al. Testing of low-risk patients presenting to the emergency department with chest pain: a scientific statement from the american heart association. Circulation. 2010 Oct;122(17):1756-76.

4 Roffi M, Patrono C, Collet JP, Mueller C, Valgimigli $\mathrm{M}$, Andreotti F, et al. ESC guidelines for the management of acute coronary syndromes in patients presenting without persistent ST-segment elevation. Task force for the management of acute coronary syndromes in patients presenting without persistent STsegment elevation of the european society of cardiology (ESC). Eur Heart J. 2016 Jan;37(3): 267-315.
5 Thygesen K, Alpert JS, Jaffe AS, Chaitman BR, Bax JJ, Morrow DA, et al. Fourth universal definition of myocardial infarction. Circulation. 2018 Nov;138(20):e618-51.

6 Apple FS, Jaffe AS, Collinson P, Mockel M, Ordonez-Llanos J, Lindahl B, et al. IFCC educational materials on selected analytical and clinical applications of high sensitivity cardiac troponin assays. Clin Biochem. 2015 Mar; 48(4-5):201-3.

7 Luepker RV, Apple FS, Christenson RH, Crow RS, Fortmann SP, Goff D, et al. Case definitions for acute coronary heart disease in epidemiology and clinical research studies: a statement from the AHA Council on epidemiology and prevention; AHA statistics committee; world heart federation council on epidemiology and prevention; the european society of cardiology working group on epidemiology and prevention; centers for disease control and prevention; and the national heart, lung, and blood institute. Circulation. 2003 Nov;108(20):2543-9.

8 Giannitsis E, Kurz K, Hallermayer K, Jarausch J, Jaffe AS, Katus HA. Analytical validation of a high-sensitivity cardiac troponin $\mathrm{T}$ assay. Clin Chem. 2010 Feb;56(2):254-61.
9 Shiozaki M, Inoue K, Suwa S, Lee CC, Chikata $\mathrm{Y}$, Ishiura J, et al. Utility of the 0-hour/1hour high-sensitivity cardiac troponin $\mathrm{T}$ algorithm in asian patients with suspected non-ST elevation myocardial infarction. Int J Cardiol. 2017 Dec;249:32-5.

10 Shiozaki M, Inoue K, Suwa S, Lee CC, Chiang SJ, Sato A, et al. Implementing the european society of aardiology $0-\mathrm{h} / \mathrm{l}$-h algorithm in patients presenting very early after chest pain. Int J Cardiol. 2020;320:1-6.

11 Ishihara Y, Shimosaka H, Kurano M, Yatomi Y, Tobita A. Evaluation of high sensitivity cardiac troponin I assay on LUMIPULSE L2400. J Jap Clin Lab Automat. 2019;44:238-44.

12 Giannitsis E. Sex-specific troponin measures for diagnosis of acute coronary syndrome. Heart. 2016 Jan;102(2):91-2.

13 Giannitsis E. Counterpoint: potential concerns regarding the use of sex-specific cutpoints for high-sensitivity troponin assays. Clin Chem. 2017 Jan;63(1):264-6.

14 Pickering JW, Greenslade JH, Cullen L, Flaws $\mathrm{D}$, Parsonage W, Aldous S, et al. Assessment of the european society of cardiology 0-hour/1-hour algorithm to rule-out and rule-in acute myocardial infarction. Circulation. 2016 Nov;134(20):1532-41. 